Prof. Dr. med. Heinrich Worth

Med. Klinik I, Klinikum Fürth
Koautor: Prof. Dr. med. Adrian Gillissen Robert-Koch-Klinik, Thoraxzentrum des Klinikums St. Georg, Leipzig

\title{
Schwangere Asthmatikerinnen oft untertherapiert
}

\begin{abstract}
Viele Schwangere haben Angst vor Medikamentennebenwirkungen. Doch eine unzureichende Asthmaeinstellung kann lebensbedrohliche Komplikationen für die Patientin und auch für das ungeborene Kind bedeuten. Heute existiert ein reicher Erfahrungsschatz zur Behandlung des Asthmas in der Schwangerschaft, der klare Therapieempfehlungen gestattet.
\end{abstract}

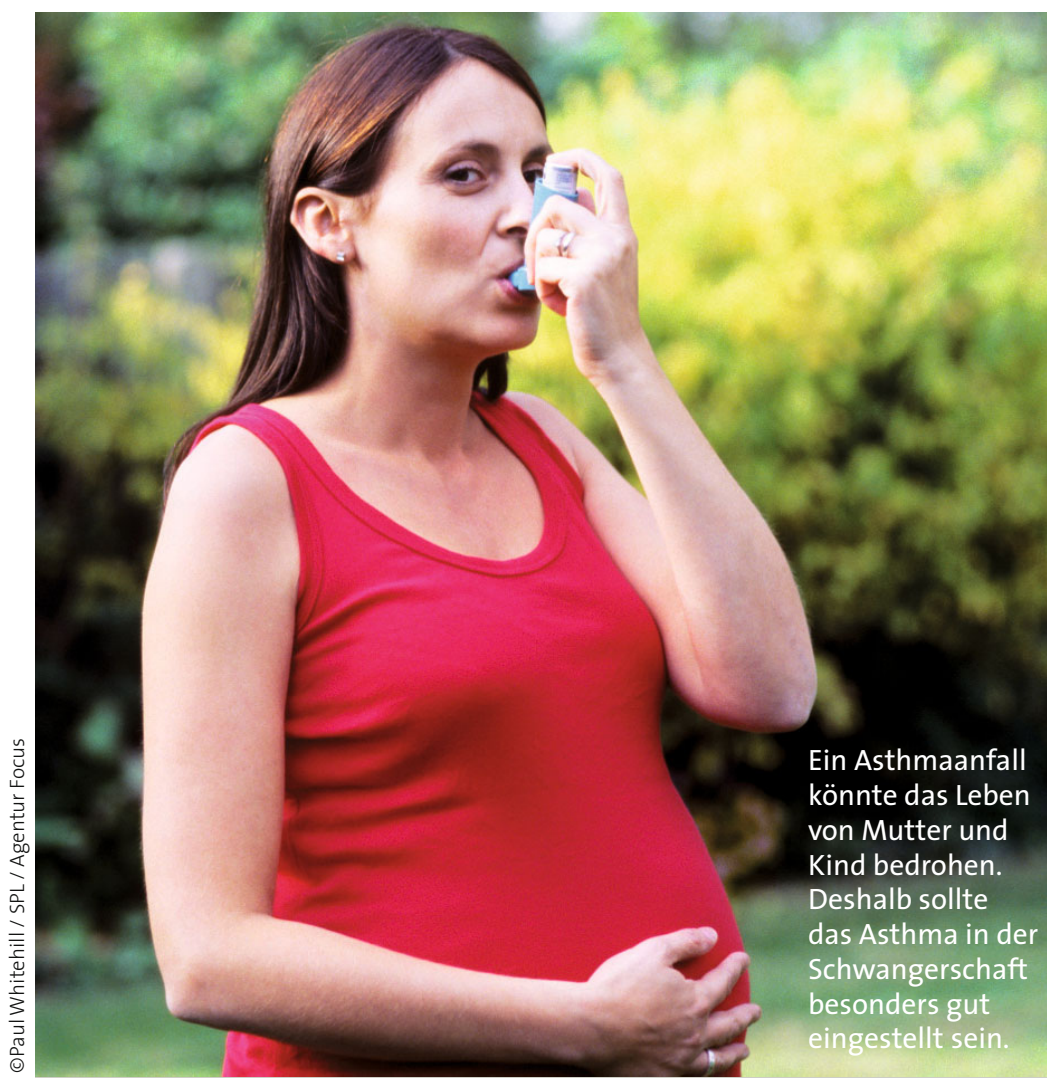

- Asthma in der Schwangerschaft birgt für den Hausarzt diverse Probleme. Es besteht z. B. Unsicherheit über mögliche teratogene Medikamentenwirkungen, die Dosierung und über den Asthmaverlauf. So verwundert es nicht, dass in einer Erhebung in Boston signifikant $(\mathrm{p}<0,02)$ weniger schwangere als nicht schwangere Frauen, die im Krankenhaus wegen eines Asthmaanfalls vorstellig wurden, mit Kortikosteroiden behandelt und mit der Empfehlung einer inhalativen Kortikosteroidtherapie wieder entlassen wurden [1]. Aufgrund der unzureichenden antiinflammatorischen Therapie war das Risiko eines erneuten, wiederum krankenhauspflichtigen Asthmaanfalls bei diesen Schwangeren um den Faktor 2,9 erhöht! Frühere Studien belegen diese letztendlich fatale ärztliche Zurückhaltung [3, 8]. Glücklicherweise besteht aber hinsichtlich der Behandlung von asthmakranken Schwangeren ein großer Erfahrungsschatz, der eine zweifelsfreie Therapieempfehlung erlaubt [4].

\section{Fötaler Sauerstoffmangel}

Ein instabiles Asthma schadet sowohl der Schwangeren als auch dem ungeborenen Kind. Der Fötus wächst ohnehin in einer Umgebung niedriger Sauerstoffspannung auf und besitzt nur eine eingeschränkte Sauerstoffreserve, so- 
dass die im Asthmaanfall auftretende Linksverschiebung der Oxyhämoglobin-Dissoziationskurve rasch zu einer kritischen Sauerstoffunterversorgung des Föten führt.

WICHTIG: Die Gefahren, die bei einer unzureichenden Asthmaeinstellung drohen, stehen in keinem Verhältnis zu den befürchteten Medikamentennebenwirkungen, die in praxi eher über- als unterschätzt werden $[7,14]$.

\section{Welche Substanzen für die Therapie? ß2-Mimetika}

ß2-Mimetika können in den zugelassenen Dosierungen bedenkenlos bei Schwangeren eingesetzt werden. Auch bei den lang wirkenden Substanzen Salmeterol und Formoterol besteht kein erhöhtes Risiko für Fehlbildungen oder perinatale Komplikationen [9]. Allerdings wurden eine geringe wehenschwächende Wirkung (OR 1,15) und eine höhere Rate an Kaiserschnitten (OR 1,79) beobachtet [5].

\section{Inhalative Kortikosteroide}

Inhalative Kortikosteroide sind die wirksamste antiinflammatorische Medikation und in der Langzeiteffizienz allen anderen Entzündungshemmern überlegen. Der frühzeitige inhalative Therapiebeginn selbst niedriger Dosen hat den besten klinischen Langzeiteffekt [13]. Fötale Missbildungen sind unter einer topischen Kortikosteroidanwendung mit einer Wahrscheinlichkeit von 1,09:1 (KI 1,03-1,15) beschrieben und daher in praxi angesichts der elementaren Bedeutung in der Asthmatherapie zu vernachlässigen $[6,12]$.

\section{Orale Kortikosteroide}

Gleiches gilt im Prinzip für die orale Kortikosteroidtherapie, obwohl über ein erhöhtes Auftreten von Kiefer- und Gaumenspalten bei oraler Kortikosteroidtherapie im ersten Trimenon berichtet wurde. Zudem können bei längerer oraler Steroidtherapie neben den bekannten Nebenwirkungen eine intrauterine Wachstumsverzögerung und eine Nebennierenrindenatrophie vorkommen. Letztere macht u. U. postpartum ein schrittweises Ausschlei-

\section{Fazit für die Praxis}

- Asthmakranke Schwangere werden in gleicher Weise therapiert wie Nichtschwangere.

- Die Chance von schwerwiegenden Medikamentennebenwirkungen ist gegenüber dem potenziellen Nutzen für die Schwangere und das ungeborene Kind vernachlässigbar.

- Der Asthmaanfall stellt für die Schwangere und ihren Fötus eine potenziell lebensbedrohliche Komplikation dar. Eine Sauerstoffgabe sowie die klinische Überwachung der Schwangeren und des Ungeborenen sind in dieser Situation obligat.

chen der Steroiddosis bei der Mutter und dem Säugling erforderlich [11] Bei einem schweren chronisch persistierenden Asthma (Schweregrad IV) sind orale Kortikosteroide in der Dauertherapie zur Verhinderung eines potenziell lebensbedrohlichen Asthmaanfalls unumgänglich.

\section{Theophyllin}

Theophyllin ist als schwächster Bronchodilatator Mittel der dritten Wahl [2]. Es gibt keine Assoziation zwischen fetalen Missbildungen oder perinatalen Komplikationen und einer Theophyllintherapie [12]. Allerdings ist Theophyllin plazentagängig, kann daher Tachykardien auch beim Fötus hervorrufen und hemmt die Uteruskontraktion. Somit gelten bei Schwangeren die gleichen Empfehlungen wie bei Nichtschwangeren.

\section{Neuere Wirkstoffe}

Wegen unzureichender Datenlage sollte eine Therapie mit dem IgE-Antikörper Omalizumab und dem Leukotrienrezeptorantagonisten Montelukast in der Schwangerschaft nicht begonnen werden. Auch bei diesen Wirkstoffen ist keine teratogene Wirkung bekannt.

\section{Asthmaanfall in der Schwangerschaft}

Die Pharmakotherapie des Asthmaanfalls einer Schwangeren entspricht der von nicht schwangeren Asthmatikerinnen (s. vorausgehenden Beitrag). Im Anfall soll unverzüglich eine Sauerstoffbehandlung eingeleitet werden mit dem Ziel, eine Sauerstoffsättigung $>95 \%$ aufrechtzuerhalten und einen arteriellen $\mathrm{CO}_{2}$-Partialdruck $<35 \mathrm{mmHg}$ $\mathrm{zu}$ erreichen.

\section{Schulung und Aufklärung}

Frauen mit Asthma, die rauchen, sollten ausdrücklich über die Gefahren des Tabakrauchens für die eigene Gesundheit (Verschlimmerung des Asthmas, Abschwächung der Wirkung inhalativer Kortikosteroide) und die des ungeborenen Kindes aufgeklärt werden. Zudem sollen ihnen Hilfen zur nicht medikamentösen Tabakentwöhnung angeboten werden.

Zur Verhinderung von Notfällen und zur Sicherstellung einer optimalen medikamentösen Therapieeinstellung müssen Schwangere umfassend über die Notwendigkeit einer kontinuierlichen Pharmakotherapie aufgeklärt werden. Die Erlernung und ärztliche Überprüfung der korrekten Inhalationstechnik für die ordnungsgemäße intrabronchiale Medikamentendeposition ist daher unumgänglich. Die Entscheidung über die Medikamentenauswahl und das am besten geeignete Inhalationsgerät (z. B. Dosieraerosol mit oder ohne Spacer, Trockenpulverinhalationsgerät) wird gemeinsam zwischen Arzt und Patientin gefällt. Mit einer strukturierten Patientenschulung können nächtliche Asthmasymptome reduziert und eine bessere Asthmakontrolle erreicht werden $[10,15]$.

\section{Literatur unter www.mmw.de}

\section{Für die Verfasser:}

Prof. Dr. med. A. Gillissen

Robert-Koch-Klinik

Thoraxzentrum des Klinikums St. Georg

Nikolai-Rumjanzew-Str. 100

D-04207 Leipzig

E-Mail: adrian.gillissen@web.de

\section{Keywords}

Bronchial Asthma in Pregnant Women

Asthma - Pregnancy - Medication Oxygen deficit - Fetus 